\title{
Neurorehabilitation Algorithm in Multiple Sclerosis: Traditions and Challenges
}

\author{
Ivet Koleva $^{1,2}$, Todor Dimitrov ${ }^{1}$ and Borislav Yoshinov ${ }^{3}$ \\ ${ }^{1}$ Department of Physiotherapy, Medical University of Sofia, Bulgaria \\ ${ }^{2}$ Department of Physical and Rehabilitation Medicine, Specialized Hospital for longtime Care and Rehabilitation "Serdika" - Sofia, Bulgaria \\ ${ }^{3}$ Medical Faculty of Sofia University, Bulgaria
}

*Corresponding author: Ivet Koleva, Medical University of Sofia, Dospat 6,

1606, Sofia, Bulgaria

Received Date: August 19, 2020

Published Date: September 08, 2020

\begin{abstract}
Multiple sclerosis (MS) is an autoimmune demyelinating disease of the central nervous system, usually beginning between 20 and 40 years (predominantly in females). Motor weakness, spasticity, balance and coordination dysfunctions provoke severe difficulty in everyday activities of MS patients. MS is a socially important disease, with a high level of acquired disability in a relatively young population. Our purpose is to emphasize the potential of some contemporary physical modalities for MS-neurorehabilitation, based on practice and research. Principal clinical and instrumental assessment and treatment methods are stated: electrical stimulations (with low and middle frequency electric currents); low frequency low intensity magnetic field; proprioceptive neuro-muscular facilitation (PNF) methods; analytic exercises, device-assisted mechano-therapy (passive, active or combined), robotic rehabilitation, etc. We insist on the importance of technical aids (wheelchair, canes, or walking sticks). Authors propose a structured Neurorehabilitation algorithm for MS-patients, with elements of physiotherapy, cryotherapy, electrotherapy, magnetotherapy, ergotherapy, diet, patient's education. We present the application of this algorithm on a typical MS-patient, treated with cryophysiotherapy, ergotherapy, electrostimulations, Locomat-rehabilitation.

Keywords: Neurorehabilitation; Multiple sclerosis; Spasticity; Expanded disability status scale; Algorithm; Functional assessment; Physical medicine; Rehabilitation

Abbreviations: ADL: Activities of Daily Life; EDSS: Expanded Disability Status scale; ES: Electrical stimulations/Electrostimulations; ET: Ergotherapy; ICD: International Classification of Diseases; ICF: International Classification of Functioning; MS: Multiple sclerosis; NR: Neurorehabilitation; PNF: Proprioceptive Neuro-muscular Facilitation; PRM: Physical and Rehabilitation medicine; PT: Physiotherapy; UEMS: Union Europeenne des Medecins Specialistes; WHO: World Health Organization
\end{abstract}

\section{Introduction}

\section{Multiple sclerosis}

Multiple sclerosis (MS) is an autoimmune demyelinating disease of the central nervous system, usually beginning between 20 and 40 years (predominantly in females). MS patients suffer from loss of autonomy and reduced quality of life, due to paresthesia, dysesthesia; motor weakness, spasticity; balance and coordination dysfunctions; depression and other signs of emotional instability or (even) cognitive impairment. Neurological guidelines consider MS as the most frequent cause of non-traumatic disability in the young adult population. These factors are closely associated to increased morbidity and mortality. It is therefore crucial that an effective treatment intervention is established, to assist the patient to manage their symptoms effectively and to remain as functional as possible [1]. The symptoms typically worsen with disease progression. MS-patients consistently report low levels of 
physical activity and poor adherence to treatment interventions. The two components, which were greatly modified in MS patients, are: muscular tone and muscular strength, components dependent on the presence or absence of muscular atrophy [2]. Multiple sclerosis (MS) presents a large heterogeneity in terms of clinical evolution, neuro-radiological images of the lesions, involvement of susceptible genes and therapy response. The "traditional" classifications of MS includes [3]: cerebrospinal, ataxic and spinal forms; with remitting-relapsing, progressive-relapsing, primary or secondary progression. Patients are transferred to a rehabilitation department with a diagnosis from an university hospital, with clinically and MRI proved MS, cerebro-spinal form, in stage of remission, with difficulties in everyday activities.

\section{Neurorehabilitation}

Rehabilitation is a functional therapy, based on a detailed functional assessment. Neurorehabilitation (NR) is an interdisciplinary field between neurology, neurosurgery, physical and rehabilitation medicine. For functional evaluation in NR we apply neurological scales and the International Classification of Functioning [4] (ICF). For treatment, we use the SMART approach (specific, measurable, achievable, realistic and timebound). We apply the "rehabilitation puzzle"- a synergic combination of different physical modalities (electric currents, magnetic field, movement, activities, temperature, etc.).

\section{Physical and rehabilitation medicine}

The number of chronic patients with diseases and conditions of the nervous and the motor systems increases and they need complex rehabilitation programs, oriented to functional recovery and amelioration of their quality of life. In this process, the impact of physical and rehabilitation medicine (PRM) is central. According the definition [5,6] of the European Union of Medical Specialists - PRM Section, Physical and Rehabilitation Medicine (PRM) is an „independent medical specialty, oriented to the promotion of physical and cognitive functioning, activities (including environment), participation (including quality of life) and changes in personal factors and environment. The specialty PRM is responsible for the management of the prevention, diagnostics, treatment and rehabilitation of patients with health-related disability and comorbidity of all ages. According the World Report on Disability [7] of the World Health Organization and World Bank rehabilitation measures are divided into three categories: rehabilitation medicine, therapy and assistive technologies. According the White Book on Physical and Rehabilitation Medicine [5,6] the basic objective of PRM is the optimization of social participation and the amelioration of the quality of life of patients. So, we must help the patient to reach possible levels and patterns of autonomy and independence, including participation in professional, social and leisure activities, part of his human rights. Tasks of PRM are: treatment of existing pathology; reduction of disability; prevention and therapy of complications; amelioration of functioning and activity; stimulation of patient's participation in different types of activities.
The World Report on Disability [7] defines the goals of rehabilitation: prevention of the loss of function; slowing the rate of loss of function; improvement or restoration of function; compensation for lost function; maintenance of current function. Modern rehabilitation has an integrative and holistic approach to the patient, based on the International Classification, disability and Health [4] (ICF) and on clinical principles. Current article presents the authors' opinion about the necessity of structuration of complex rehabilitation algorithms (PRM programmes of care), including not only different natural and pre-formed physical modalities, but too a detailed functional evaluation at the beginning (baseline) and at the end of every NR-course - in all neurological patients.

\section{Functional Assessment in MS}

The first step of our algorithms is the qualitative and quantitative evaluation, based on scales, applied commonly in the clinical practice of neurology and rehabilitation. The functional assessment of patients with neurological conditions, with sensory, motor, autonomic dysfunctions and deficiencies must be effectuated before and after every rehabilitation course. The evaluation have to be based on the holistic approach to the patient, including diagnosis according the International Classification of Diseases [8] (ICD - diagnosis); specialized neurological \& orthopedic examination; detailed functional assessment; ICF-assessment [4] (ICF, 2001). In Bulgarian neurorehabilitation practice we apply traditionally the Expanded Disability Status Scale (EDSS - Kurtzke) and (in some cases) the Barthel-index. According ICF principles [4] the complex functional assessment must include: body functions (range of motion, muscle force or motor deficiency, alterations of coordination - ataxia, intentional tremor, spasticity; irritative sensory signs); activities (verticalization, mobility, grasp, standing up, gait, activities of daily living (ADL), transport); participation (family relationship, relaxing activities, social life, political activity); environmental factors (conditions of life and work, transport, family and friends, health insurance, social relationship); personal factors (life style, co-morbidities, age, sex).

During clinical assessment we accentuate on some analyses [9-11]: pain (localization, type, intensity - verbal or visual analogue scale); joint stability (including joint position sense) and range of motion (active and passive); presence of muscle or joint contractures; evaluation of the muscle force / muscle insufficiency, motor deficit; analysis of the grasp and gait; mobility (necessity of technical aids: canes, walking sticks, crutches, walkers, wheelchairs or other devices); fatigue (physical endurance, necessity of rest during the examination or the functional activity); autonomy in everyday activities (bathing, dressing, eating, putting shoes on, personal hygiene, need of help in activities of daily life - ADL). Evaluation of problems must be qualitative and quantitative, including: fatigue, motor deficiency, coordination problems (body position, gait, grasp); irritative sensory signs and pain; conscience for the necessity of technical aids and assistive technologies; difficulties in ADL; limitations of functional mobility. 
The control before and after neurorehabilitation is obligatory. At the end of every course we realize a detailed clinical, para-clinical and functional (including instrumental) revision of the obtained results, and we prescribe a periodical control and periodical PRM courses. We consider that the functional evaluation is very important not only for control of the quality of rehabilitation, but too for amelioration of independence in everyday activities and of health-related quality of life of patients.

\section{Physical Modalities, Applied in MS Neurorehabili- tation}

In clinical NR-practice [9], many traditional and contemporaneous physical modalities and methods are applied:

- $\quad$ From the field of physiotherapy [12,13]: proprioceptive neuro-muscular facilitation (PNF) techniques; aerobic exercises; strengthening exercises, analytic exercises; stretching and range-of-motion exercises; bimanual and bipedal training (if possible); Regular physical activity (repetition of exercises with mild to moderate intensity); grasp training [14] and Mirror therapy; balance and gait training [14]; device-assisted mechanotherapy (passive, active or combined);

- From the domain of ergotherapy (occupational therapy): Education of the person with disability trough task oriented (task-specific) activities; Art therapy, music-therapy; relaxation techniques (including sleeping); technical aids (wheelchair, canes, or walking sticks); home adaptations;

- $\quad$ From the field of balneotherapy: cryotherapy; underwater exercises; etc.

- From the field of preformed physical modalities: electrical stimulations [15] (with low and middle frequency electric currents); transcranial and transmedullar magnetic stimulation; deep oscillation; etc

Table 1: Parts of the complex neurorehabilitation programme [9].

\begin{tabular}{|c|c|c|c|c|}
\hline $\begin{array}{c}\text { KINESI- and ERGO } \\
\text { therapy } \\
\text { (exercises, activities) }\end{array}$ & $\begin{array}{c}\text { PREFORMED Modalities } \\
\text { (electrostimulations, magnetic field) }\end{array}$ & $\begin{array}{c}\text { CRYO-/ HYDRO-Therapy } \\
\text { (ice, mineral waters) }\end{array}$ & $\begin{array}{c}\text { DIET } \\
\text { (amino-ac- } \\
\text { ids) }\end{array}$ & PATIENT EDUCATION \\
\hline
\end{tabular}

In our clinical practice, we apply a synergic combination of two procedures with pre-formed modalities (electrostimulations; magnetic field); one cryo- or hydrotherapeutic procedure with three kinesi-therapeutic methods and minimum one ergotherapeutic activity. For the structuration of the individualized NRprogramme we used everywhere capacities of different traditional and contemporaneous natural and pre-formed factors, accentuating on the potential of modern methods, e.g.: electrostimulations (FES), proprioceptive neuro-muscular facilitation, analytic exercises, postisometric relaxation, stretch techniques; robotic NR, Exoskeleton, virtual reality rehabilitation; and ergo-therapeutic methods. The functional recovery and the autonomy depend on the intensity of the grasp and gait-training. We consider that in MS-cases with important spasticity cryotherapy is obligatory part of the
Robotic NR: Exoskeleton, Locomat, Virtual reality, etc.

\section{Neurorehabilitation Programme in MS}

The complexity of neurorehabilitation in MS imposes the necessity of a holistic approach to the patient - detailed functional analysis before and after the NR-courses; application of therapeutic methods of different medical specialties (principally neurology; orthopedics; rheumatology; PRM) and from nonmedical fields (kinesi / physio-therapy, sociology, psychology, occupational therapy). We use the SMART approach (specific, measurable, achievable, realistic and timebound); according the basic principles of the specialty Physical and Rehabilitation medicine [5,6]. Depending on the results of the assessment of the rehabilitation potential of the concrete patient, we apply different physical modalities and methods in different combination - the rehabilitation puzzle. In every stage of the rehabilitation processus we must define precisely the goal, tasks and algorithms of NR. In every case our goal is to assure a high quality of the NR-programme and procedures, optimal for the clinical form of the principal disease or condition, adapted to the age, co-morbidities, capacity and preferences of the concrete patient; with the strategic goal to receive the best result for his quality of life.

Many NR tools have the potential to rewire cerebral functions and to excite the formation of new connections and pathways, respectively to stimulate the brain reorganization and adaptation to the 'new' situation (appearance of a damaged locus in the cerebral tissue), in other terms - to help functional recovery through potentiation of use-induced and use-dependent neuroplasticity. The NR-puzzle is a synergic combination of different physical modalities (electric currents, magnetic field, movement, activities, temperature, etc.). The complex NR-programme includes physical and drug therapy, diet, patient education (Table 1).

programme. In MS-cases with significant fatigue we must divide the physio-therapy, ergotherapy and robotic training into several parts of 15 or 20 minutes everyone. The presented PRM algorithm can be concretized and we used it during our own clinical investigations and observations (including clinical case studies) of MS-NR.

\section{Clinical Example of the Structuration of the NR- Programme for a typical MS-Patient}

We present a case of a female of 40 years, MS - cerebrospinal type, relapsing-remitting form with secondary progression, duration of the disease 10 years. The diagnosis MS is clinically and MRI proved (Figure) in a Neurological Clinic of the University hospital (Figure 1). 
The patient was transferred to our PRM-Department 15 days after a relapse. At the admission, she presented a quadriparetic syndrome with inferior spastic paraplegia; cerebellar ataxia (static ataxia in sitting position, dynamic dyscoordination); urinary incontinence (urinary catheter). Spasticity - 3+. Pathologic reflexes of the Babinski and Rossolimo groups in feet. Defensive reflexes in lower extremities. Clonus of the right foot. Wheeled mobility (using an electric wheelchair). EDSS-score: 7,5 at the admission. In the NR-complex we included: cryotherapy, physiotherapy, ergotherapy, electrostimulations for the feet extensors, LOCOMAT-rehabilitation (Figure 2).

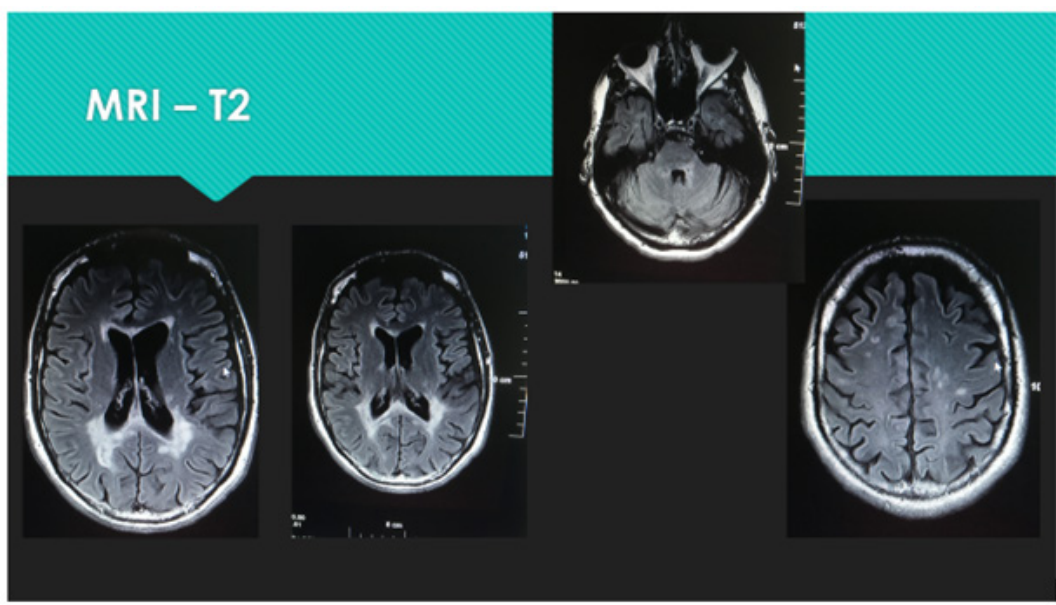

Figure 1: MRI of our patient.

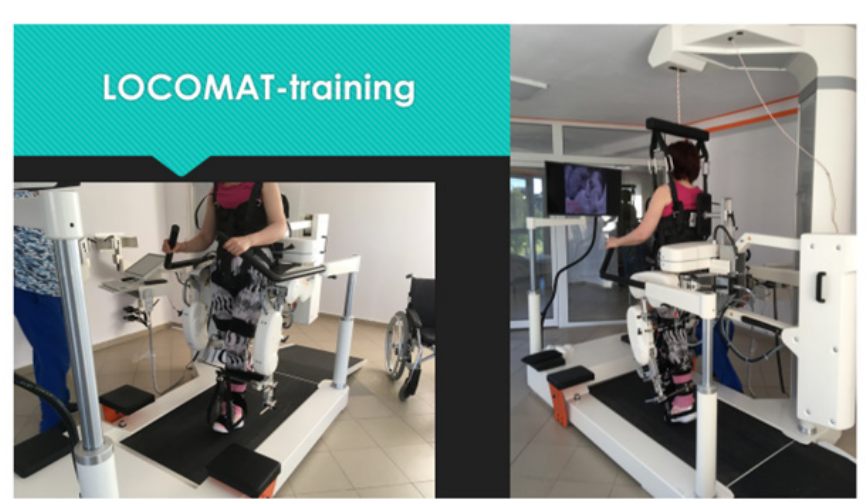

Figure 2: Gait training with LOCOMAT of our patient.

The control was done before, during and at the end of the NR course (of 30 treatment days), and one month after its end - using a battery of clinical methods and functional scales: Posture, Trunk Control, Balance, Transfers; Ambulation/Mobility; goniometric evaluation of the Range of Motion (ROM) of knees and ankles; Motor Function in upper and lower extremities; Neurologic exam spasticity, pathologic reflexes, clonus, sensation, pain; coordination assessment (Romberg, dysdiadochokinesis test, marionettes test); grasp assessment; intentional tremor assessment. Respiratory function; Wheeled mobility. During autonomy assessment we accentuated on self-service activities, mobility and transport capacities.

The complex NR-programme, included everyday séances of many procedures, as follows:

- $\quad$ cryotherapy (ice-packages) for the spastic muscles (m.triceps surae, m. tibialis anterior) and for ankle contractures.
- Antagonists' electrostimulations - for feet extensors (m.tibialis anterior, m.peroneus longus, m.extensor digitorum longus, m.extensor halluces) - galvanic current, tetanic pulses, 10-15 minutes, 20 procedures.

- $\quad$ proprioceptive neuromuscular facilitation techniques; aerobic exercises and general strengthening for arms and legs; analytic exercises;

- bimanual coordination exercises;

- $\quad$ stretching of ankle ligaments and exercises for ankle ROM;

- $\quad$ Relaxation techniques and Yoga elements

- $\quad$ Grasp training and Goal-oriented activities (for ADL);

- $\quad$ Training of the Posture, Balance \& Trunk control (in sitting position), 
- $\quad$ gradual verticalization, balance and gait training with two crutches and two physiotherapists.

- $\quad$ Coordination, transfers and ambulation (gait) training;

- $\quad$ Balance and gait training with LOCOMAT.

The physiotherapeutic procedures and the Locomat séances were fractioned into 4 times per day, due to fatigue and increase of spasticity.

We observed amelioration in trunk control, range-of-motion of ankle joints and decrease of motor weakness of lower limbs, reduction of spasticity, amelioration of the balance; improvement of functional capacity and autonomy in ADL.

\section{Discussion}

\section{Efficacy of NR-algorithms in clinical practice}

During our modest clinical practice (of 30 years) in the NRfield, we observed the efficacy of NR on the functional recovery and the autonomy of neurological patients. The goal of our work was to prove and evaluate the efficacy of application of different modalities and NR-methods on independence and quality of life of neurological patients. We effectuate a composition, clinical application and approbation of complex NR-algorithms. Patients were divided into many groups and subgroups, in each one we applied a different NR-complex, composed by a synergic combination of natural and pre-formed physical modalities (electrical currents, laser; cryoagents, hydro-therapy; physiotherapy and occupational therapy). Patients were controlled before, during and at the end of the NRcourse and one month after its end - using a battery of traditional and contemporaneous evaluation methods: tests and scales for motor deficiency, balance and coordination; functional grip of the upper limb; balance, gait and independent motion; independence in activities in daily living (ADL: self service, family, professional and social life); depression and anxiety; visual analogue scale of pain; vibroesthesiometry; thermosensibility; ICF asessment. Based on detailed qualitative and quantitative evaluation we proved the efficacy of application of different NR-programmes - on different types and levels of sensory, motor and functional deficiency.

Our opinion is that the role of different elements of the complex NR-programmes must be considered for stimulation of functional recovery and for improvement of everyday quality of life of MS patients.

The on-time initiation of rehabilitation procedures in neurological management demonstrates many positive results: amelioration of the clinical status of patients and prevention of complications; decrease of muscle weakness, increase of muscle force and range of motion, pain reduction, regulation of the balance / equilibrium; normalization of the muscle dysbalance and neuromuscular coordination; functional recovery of the grasp and the gait; amelioration of autonomy and quality of life of patients; acceleration of the resocialization and inclusion in functional activity, economic effect.

\section{Neuroplasticity and neurorehabilitation}

According Merriam Webster dictionary: "neuroplasticity is the adult brain's ability to adapt". According the medical dictionaries: "neuroplasticity is the brain's capacity to reorganize itself by forming new neural connections throughout life" [16]. Neuroplasticity allows the neurons to compensate for injury and disease and to adjust their activities in response to new situations or to changes in their environment. Principal mechanisms of brain repair are based on brain plasticity (spontaneous recovery, input of "axonal sprouting" and "mirror-neurons", use-dependent plasticity, synaptic or grey matter plasticity, white matter plasticity).

For patient adaptation to the "new" situation (of neuronal alteration), we use two types of modulation techniques: stimulation of some functions (e.g. motor and sensory functions) and inhibition of other (e.g. pain reduction). For stimulation, we apply training of the altered motor function (through movements and activities - use-dependent plasticity) and some compensatory mechanisms (bypass strategies, replacement of functions by aiding devices, adaptation of the environment to patient's needs). For inhibition, we apply pain management mechanisms: blocking the nociception and the neurotransmission, peripheral sympaticolysis, input the gate-control, peripheral and central desensitization, influence on the descending systems for pain control and activation of the encephalic blocking system of the central nervous system, activation of reflectory connections.

The "aim" of neuroplasticity is to optimize neural networks during phylogenesis, ontogenesis and physiological learning, and in case of a brain disease.

Neuroplasticity is the pathophysiological basis for treatment of the cerebral lesions through physical training and rehabilitation, including goal-directed activities. Rehabilitation is a functional therapy, based on a detailed functional assessment. Practically, every rehabilitation process is founded on neuroplasticity (training or use-dependent plasticity). Based on our modest clinical practice (of 30 years) in the NR-field and our own results, we emphasize on the capacity of physical modalities for stimulation of neuroplasticity (especially on activity-induced plasticity), on the potential for functional recovery and amelioration of independence in everyday life of patients with diseases and conditions of the nervous systems.

\section{Neurorehabilitation team}

For effective gait rehabilitation the inclusion of a multiprofessional therapeutic and rehabilitation (neurorehabilitation) team is obligatory. Different models of organization of the teamwork of the staff are applied: interdisciplinary (complex care of the patient from different scientific and professional disciplines); multi-disciplinary (role of every professional is completely independent from the others); transdisciplinary (everyone helps the work of the others; role and functions are distributed). 
We consider that the clinical practice imposes the necessity of transition from a multi-disciplinary to a transdisciplinary model of team work, with a clear definition of the fields of competence and the responsibility of the team members. In Bulgarian rehabilitation practice traditionally a lot of specialists are included: medical doctors - specialists in Neurology, Neurosurgery; Rheumatology; Orthopedics and Traumatology and in Physical and Rehabilitation Medicine (PRM); bachelors and masters in Physical Therapy and in Occupational therapy (Kinesio-therapy and Ergo-therapy according nomenclature of our country).

\section{Conclusion}

In our clinical practice we apply systematically our structured NR-algorithms, individualized and adapted to the concrete patient. We published periodically our results $[9,14,15]$, proving the amelioration of the quality of life of many MS patients. In case of synergic combination of procedures, we received statistically significant favorable effects on the range of motion, muscle force and muscle weakness, neuro-muscular coordination, grasp and gait, independence in ADL, quality of life of patients. Authors consider that NR must be included in MS-therapeutic guidelines. Complex neurorehabilitation stimulates functional recovery of patients with invalidating diseases and conditions of the nervous system, ameliorating their independence and the health-related quality of life.

\section{Acknowledgement}

We express gratitude to our staff of all the PRM-Departments and Clinics - base of our work. The coordinated teamwork is the crucial circumstance of the successful Neurorehabilitation.

\section{Conflict of Interest}

Authors declare the non-existence of any financial interest or any conflict of interests.

\section{References}

1. Pidgeon L, Pelton T, Soundy A (2017) Determining the use and value of social support in Telerehabilitation Interventions for individuals with Multiple Sclerosis: A narrative synthesis review. J Nov Physiother Rehabil 1: 120-136.

2. Neamţu MC, Rusu L, Rusu PF, Neamţu OM, Georgescu D, et al. (2011) Neuromuscular assessment in the study of structural changes of striated muscle in multiple sclerosis. Rom J Morphol Embryol 52(4): 1299-1303.
3. Confavreux C, Vukusic S, Moreau T, P Adeleine (2000) Relapses and progression of disability in multiple sclerosis. N Engl J Med 343: 14301438.

4. World Health Organization (2001) International Classification of Functioning, Disability and Health (ICF). - WHO, Geneva.

5. White Book on Physical and Rehabilitation Medicine in Europe (2007) Produced by the Section of Physical and Rehabilitation Medicine, Union Europeenne des Medecins Specialistes (UEMS), the European Board of Physical and Rehabilitation Medicine and l'Academie Europeenne de Medicine de Readaptation in conjunction with the European Society of Physical and Rehabilitation Medicine (ESPRM). In: C Gutenbrunner, AB Ward, MA Chamberlain (Eds.), Journal of Rehabilitation Medicine 1(45): $1-48$.

6. White Book on Physical and Rehabilitation Medicine in Europe (2018) European PRM Bodies Alliance: European Academy of Rehabilitation Medicine, European Society of PRM, EUMS - PRM Section, European College of PRM. European Journal of Physical and Rehabilitation Medicine 54(2): 1-204.

7. World Health Organization and the World Bank (2011) World Report on Disability. - Geneva: WHO Press.

8. World Health Organization (2018) International Classification of Diseases (ICD - revision XI)- Geneva, WHO.

9. Koleva I Editor (2019) Clinical Neurorehabilitation (electronic monograph). - Sofia: SIMEL, 678 pp. SIBN: 978-619-183-085-5

10. Bethoux F, Calmels P (2003) Guide de mesure et d'évaluation en médecine physique et de réadaptation. - Paris: Roche.

11. Stucki G, Ewert T, Cieza A (2002) Value and application of the ICF in rehabilitation medicine. Disability and Rehabilitation 24: 932-938.

12. DeLisa JA (2005) Physical Medicine and Rehabilitation - principles and practice. 4th Edition. - Philadelphia: Lippincott, Williams \& Wilkins.

13. American Academy of Physical Medicine and Rehabilitation Task Force on Medical Inpatient Rehabilitation Criteria (JL Melvin Chair). (2006) Standards for Assessing Medical Appropriateness Criteria for Admitting Patients to Rehabilitation Hospitals or Units. [Accessed March 6, 2008] Available at: http://www.aapmr.org/ hpl/legislation/mirc.htm

14. Koleva I, Avramescu T Editors (2017) Grasp and Gait rehabilitation (Bases). Monograph. - Sofia: 'SIMEL Press', 396 pages. Printed version: ISBN: 978-619-183-055-8.

15. Koleva I, Voukadinova E, Yoshinov B (2019) Impact of functional electrostimulations in the complex neurehabilitation algorithm of patients with multiple sclerosis. Journal of Physiotherapy \& Physical Rehabilitation 4(54): ISSN: 2573-0312.

16. Didier J (2004) La plasticité de la fonction motrice. Collection de l'Académie Européenne de Médecine de Réadaptation. - Paris: Springer Verlag, $476 \mathrm{pp}$. 